\title{
The Anesthesia and Coping Strategies of Medical Care for Astronauts in Shenzhou Eleven Spaceship
}

\author{
Liu Ji, Fang Weiwu, Jia Fukong, Song Haifeng, Lou Xiaotong, Li Lu, Yang Heming, Tan Rong, \\ Shi Tiejun, Wu Jie, Zhou Li, Wang Yuan, Gu Jianwen*
}

The Strategic Support Force Medical Center of PLA, Beijing, China

Email address:

liuji_school@163.com ( (Liu·Ji),2914081083@qq.com (Gu Jianwen)

${ }^{*}$ Corresponding author

\section{To cite this article:}

Liu Ji, Fang Weiwu, Jia Fukong, Song Haifeng, Lou Xiaotong, Li Lu, Yang Heming, Tan Rong, Shi Tiejun, Wu Jie, Zhou Li, Wang Yuan, Gu Jianwen. The Anesthesia and Coping Strategies of Medical Care for Astronauts in Shenzhou Eleven Spaceship. Clinical Medicine Research. Vol. 8, No. 5, 2019, pp. 119-124. doi: 10.11648/j.cmr.20190805.15

Received: September 9, 2019; Accepted: October 14, 2019; Published: October 23, 2019

\begin{abstract}
To analyze the characteristics and basic principles of the medical support and anesthesia for the Shenzhou eleven astronaut landing field, and to summarize the experience of the medical care and anesthesia in the main landing field of our country, and to ensure the security tasks in the special environment such as the emergency return of manned space flight. To review the reports on the medical care and anesthesia of the astronauts at landing field, and summarize the experience of the medical care and anesthesia of the Shenzhou five to the Shenzhou ten astronaut medical support landing field, and put forward a series of organizations and prepositions on the environment characteristics of the "Shenzhou eleven" in the long time and the cold weather in the main landing field of the scheduled return time. Case, equipment and effective treatment plan, and be tested in actual combat. On the basis of the original first aid, the first aid equipment was adjusted, the first aid procedure was optimized, the heat preservation heating device was added, the laryngeal mask, the anti fog visible laryngoscope and the portable ultrasonic device were introduced, and the anesthesia and medical care plan for the manned space landing field was formulated, which was the astronaut stay in the space and the cold and complicated weather conditions. The treatment and medical care provided a strong guarantee. The first aid support scheme of the Shenzhou eleven spacecraft's main landing field was improved and optimized, and it fully met the medical security task of the special environment such as the astronaut emergency return and so on in the cold weather.
\end{abstract}

Keywords: Astronaut, Anesthesia, First Aid, Medical Rescue, Medical Security

\section{Introduction}

The Shenzhou 11 spacecraft is the first manned spacecraft to return in winter in China's manned space history. The two astronauts spent 33 days in orbit, creating a new record of Chinese astronauts' time in space. The medical rescue team of the main landing site of manned spaceflight in PLA 306 Hospital consulted the relevant reports of first aid and resuscitation of astronauts at home and abroad, summarized the experience of medical security for astronauts from Shenzhou V to Shenzhou 10, analyzed the flight characteristics of Shenzhou 11, and put forward a series of medical rescue and protection countermeasures. Strategies, successfully completed the medical security and rescue mission. This article tries to summarize the experience of medical security and rescue from the angle of anesthesia $[1,3$, 5, 7, 10, 24-27].

\section{Significance and Current Status of Space Anesthesiology}

With the vigorous development of manned spaceflight in China, medical support for astronauts is inevitably involved. National Aeronautics and Space Administration (NASA) experts estimate that the risk of serious medical incidents in space exploration missions is 0.06 per flight year [1]. In fact, emergency surgical treatment of severe trauma after landing means that the ability to anesthetize immediately after landing is required [2].

The implementation of anesthesia rescue in landing site 
must take into account the special effects of weightlessness environment on physiology and the anesthesia risk. The tragedy of death and serious anesthesia-related complications of two rhesus monkeys on Bion 11 Biological Experimental Satellite during anesthesia after landing suggests that our knowledge of space anesthesia is incomplete [3]. Up to now, human beings have entered the field of manned space for more than 50 years, and no anesthesia has been carried out by human astronauts in the course of space flight or after landing for emergency medical treatment or research purposes.

\section{Anesthetic Physiological Effects of Shenzhou 11 Astronauts After Their Mid-term Residence}

The emergency return of astronauts refers to the normal return of the spacecraft to the state where the astronauts are injured or sick or the spacecraft returns informally. Emergency return of spacecraft significantly increases the risk of astronauts entering emergency medical procedures for surgery and anesthesia. The on-orbit flight time of Shenzhou 11 is 33 days. The prolonged flight time leads to the failure of the spacecraft, the increased risk of the astronauts' on-orbit illness and the increased possibility of emergency return. Exposure to microgravity for a long time has brought about a series of changes in astronauts' cardiovascular circulation, airway, stress response and other physiological conditions.

\subsection{Cardiovascular System}

The cardiovascular system is undoubtedly the most critical part of emergency medical care during or after space flight from the perspective of anesthesiologists.

\subsubsection{Fluid Transfer and Reduction of Effective Circulating Blood Volume}

Entering the weightlessness environment, the hydrostatic pressure dependent on gravity disappears. In the lower extremities, body fluid transfers from extracellular space to intravascular space, while in the head of the body, it transfers from intravascular space to extracellular space. Astronauts experience head and face swelling, nasal congestion, scleral and oral mucosal edema, and lower limb circumference reduction, a phenomenon known as "fluid transfer". In weightlessness environment, oral intake of diet decreases, while body fluids transfer to intercellular matrix and cells, which leads to the reduction of effective blood volume. On the first day of entering the weightlessness environment, the blood volume of human body decreases by about $9-17 \%$. If the body weight is $70 \mathrm{~kg}$, it is equivalent to $1 \mathrm{~L}$ blood volume reduction [4-6].

\subsubsection{Decreased Cardiac Output, Anemia and Arrhythmia}

Head-to-head transfer of body fluids results in a $20-50 \%$ increase in stroke volume and cardiac output. In weightlessness environment, the decrease of blood volume and myocardial atrophy leads to the decrease of stroke volume, and the decrease of cardiac output can reach $17-20 \%$. As the end-diastolic volume decreases more than stroke volume, ejection fraction increases. Under weightlessness, the number of red blood cells decreased by about $10 \%,[10]$ may be related to the decrease of erythropoietin secretion. In space, especially during spacewalk, the risk of arrhythmia increases significantly, which may be related to the increased release of catecholamines $[8,9]$.

\subsubsection{Decreased Sensitivity of Adrenergic Receptors and Baroreflex}

Under weightlessness, the sensitivity of beta-adrenergic receptor increased and that of alpha-adrenergic receptor decreased. Long-term space flight ( $>14$ days) inhibits baroreceptor reflex, which disappears about two weeks after landing on Earth [3, 11]. These effects have guiding significance for anesthesia rescue in landing site.

Changes of vascular endothelial cells and vascular remodeling and reduction of peripheral vascular resistance.

Under weightlessness, vasodilation and systemic vascular resistance decreased by $14+9 \%$. [4, 12] NASA astronauts used a 4-F diameter catheter to measure the central vein in weightlessness environment. They found that the central venous pressure did not increase, but decreased [13]. It may be due to the disappearance of negative pressure in diastole and pleural cavity under weightlessness [14].

In the tail suspension simulated weightlessness model of rats, it was found that weightlessness caused changes in vascular endothelial cell function, suggesting that the occurrence of vascular endothelial dysfunction under weightlessness may be related to endotoxin translocation in gastrointestinal tract. Under weightlessness, the transmural pressure of microcirculation changes. Vascular endothelial cell dysfunction and changes in microcirculation transmural pressure may lead to vascular remodeling $[15,16]$.

Vascular remodeling and decreased vascular tension under weightlessness may be the cause of orthostatic intolerance after landing $[15,16]$.

\subsection{Respiratory System}

Compared with the measurements before space flight, the breathing frequency increases by about $9 \%$ and the tidal volume decreases by about $15 \%$ in space. As a result, alveolar ventilation volume did not change significantly, and functional residual air volume increased. This theoretically reduces the risk of atelectasis after mechanical ventilation. Under weightlessness, the ratio of ventilation to blood flow is more ideal, which is helpful to improve oxygenation. The increase of alveolar blood flow contact area is beneficial to the increase of lung diffusion. Parabolic flight studies have shown that changes in thoracic and abdominal compliance are fundamentally beneficial to lung function. Intra-abdominal lesions and elevated intra-abdominal pressure can impair lung function, which has a significant impact on advanced life support, especially ventilation management [17-19].

\subsection{Musculoskeletal System and Acetylcholine Receptor}

Under weightlessness, skeletal muscle atrophy and bone loss 
occur. This has a significant impact on astronauts to complete space missions, and is also the current research hotspot. There is no relevant study on muscle relaxants given to astronauts with skeletal muscle atrophy under weightlessness. Therefore, anesthesia induction may be a wise choice for ICU patients or patients with myasthenia who have been in bed for a long time. For such patients, intravenous injection of succinylcholine may lead to hyperkalemia and cardiac arrest. Weightlessness affects the structure and distribution of acetylcholine receptors, which may lead to hyperkalemia during succinylcholine injection $[2,7$, 20].

It is very important for anesthesiologists to obtain data on the use of depolarized and non-depolarized muscle relaxants in weightlessness. Before obtaining these data, a reasonable induction method should be to give sufficient induction time and dose-by-dose non-depolarizing muscle relaxants to observe their effects. In addition, non-depolarizing muscle relaxants such as rocuronium should be used if rapid sequence induction is required $[7,20]$.

\subsection{Other Systems}

Under weightlessness, the immune function was reduced and the incidence of infectious diseases was increased. Changes in bacterial virulence in space can further increase the likelihood of infection [21].

After 72 hours of space flight, gastrointestinal activity and gastric content $\mathrm{PH}$ decreased. Astronauts usually show weight loss, with an average weight loss of about $5 \%$ after six months at the International Space Station (ISS) [22]. This is mainly due to the imbalance between calorie intake and consumption.

\section{Analysis of Anesthesia Rescue Procedures and Challenges in Emergency Return of Astronauts}

\subsection{Anesthesia Rescue Procedure}

The medical rescue team divides the anesthesia rescue after landing into three stages, and formulates the anesthesia rescue plan, namely the basic life support at the landing site of the return capsule, the advanced life support at the helicopter transit stage, and the anesthesia induction and comprehensive treatment at the hospital or designated medical institution during transit.

\subsection{Consideration of Anesthesia}

There are many factors affecting anesthesia after landing, which can be roughly divided into the following two categories: medical equipment factors and aerospace anesthesiology related knowledge factors.

\subsubsection{Medical Equipment}

Advanced medical care usually requires a specific set of equipment, such as monitors, ventilators, sputum suction equipment and compressed oxygen storage equipment. However, the carrying space of field equipment resources is limited, and the means of judging and diagnosing injuries are lacking. It is difficult to obtain medical data management and aseptic environment.

\subsubsection{Risks of Lack of Relevant Knowledge}

The gravitational environment of the ISS is $12 \%$ or totally weightless of the Earth, the surface gravity of the Moon is $1 / 6$ of that of the Earth, and Mars is 1/3 of that of the Earth. What is the minimum gravity level to prevent the body from functioning downward? Accurate knowledge of human adaptation to partial weightlessness environment is crucial to health care.

Cardiovascular balance re-established after adaptation in weightlessness environment is fragile, and tolerance to any accident (such as loss of blood loss, allergic reaction or reduced cardiac function) or interventional therapy (such as general anesthesia or mechanical ventilation) may be reduced.

The changes of body weight, fluid redistribution and renal perfusion under weightlessness will affect the pharmacodynamics and pharmacokinetics of drugs.

\subsubsection{Selection of Anesthesia Regimen Through Literature Review}

Astronauts have very limited experience in emergency medical procedures after landing. McSwain et al. argued in his written communication that safe anesthesia is possible with a full understanding of the physiological changes in human space.

In the absence of strong evidence, it is advisable to formulate anesthesia options for the worst cases (e.g., the effects of cardiovascular down-regulation on general anesthesia under weightlessness, astronauts' shock, arrhythmia, difficult intubation, satiety of stomach, and inability to use succinylcholine for rapid sequence induction).

The risk of local anesthesia is very small, but it requires a lot of training, whereas general anesthesia is the opposite. The application of ultrasound greatly simplifies the difficulty of local anesthesia and enhances the safety and success rate of local anesthesia.

Spinal anesthesia and epidural anesthesia can also be considered. However, its effect and safety may be unpredictable after weightlessness, because fluid transfer and downregulation of cardiovascular function will make anesthesia management difficult, and require a lot of training and sterilization.

General anesthesia is suitable for any situation. Fluid transfer and head and face edema after weightlessness may increase the risk of difficult intubation. Muscle relaxants are recommended for general anesthesia to facilitate tracheal intubation. Astronauts should undergo an allergy test before flying in space. The application of video laryngoscope and laryngeal mask can improve the success rate of tracheal intubation and mechanical ventilation

\section{Results}

\subsection{Astronaut Exit Process Optimization}

Based on the experience of the past five manned flight landing sites and combined with training exercises, the medical team optimized the "Shen 11" exit plan, that is, medical supervisors and medical insurance personnel enter the 
return capsule and complete the necessary work, immediately exit the capsule.

\subsection{Establishment of an Efficient Ambulance System}

The medical emergency rescue work of manned space flight is a complete system engineering. It is necessary to continuously strengthen and improve the medical rescue system of landing site, which is the key measure to ensure the success of rescue.

The specific practice of the medical team is that the medical supervision and insurance medical rescue team of the main and auxiliary landing site is equipped with manned spaceflight medical ambulance, medical helicopter and a full set of advanced portable first aid equipment, so as to achieve one, two, three, four implementation. The medical rescue team of Shenzhou 11 main landing site is composed of eight specialists, divided into two groups, each group of four people, corresponding to two astronauts. Experts include emergency anesthesia, general surgery, cardiothoracic surgery, orthopaedics, neurosurgery, cardiovascular medicine, nursing, etc. Each group is equipped with a helicopter rescue platform, including advanced and complete basic life support equipment, advanced life support equipment, to achieve ICU rescue capabilities, and have the ability to conduct damage control surgery. Medical rescue helicopter has advanced communication equipment, can timely understand the astronauts' information, once there is an abnormal situation, can be prepared for treatment as soon as possible. In order to ensure the efficient and successful pre-cabin treatment, each medical team is equipped with a complete set of first aid equipment, including advanced and complete basic life support equipment [23-26], has the ability to independently rescue astronauts.

Because the landing probability of Shenzhou spacecraft in the main and sub-landing sites is the highest, medical rescue teams and mobile ICU platforms are equipped for the main and sub-landing sites, standardized procedures are implemented, and repeated drills are carried out on this basis.

\subsection{Implementation Plan of Anesthesia Rescue in the Main Landing Site of Shenzhou 11 Spacecraft}

\subsubsection{Principles of Anesthesia Rescue}

The principle of medical treatment for emergency return of astronauts is that when astronauts are injured or sick, medical rescuers arriving at the scene should implement necessary and urgent medical treatment for astronauts. After on-site medical treatment is completed, medical supervisors, medical insurance and medical rescue personnel are jointly responsible for transferring astronauts to back branch hospitals or designated medical institutions for comprehensive diagnosis and treatment or medical examination.

\subsubsection{Basic Life Support}

\section{$i$. Break out the spacesuit for chest compression}

Extracorporeal cardiopulmonary resuscitation (CPR) chest compression for astronauts requires the first removal of spacesuits to expose astronauts. For the complex structure of spacesuit, in order to break down the spacesuit, it is necessary to take the astronaut's lateral decubitus position with the cooperation of medical supervision and medical insurance personnel and medical rescue personnel, and break the spacesuit from the back with professional equipment.

ii. Airway opening and ventilation

During the simulation exercise of Shenzhou 11 medical rescue and support mission, the medical team adjusted the list of materials according to the influence of cold weather on equipment and materials and the prediction of airway opening and mechanical ventilation after astronauts' mid-term stay in space, replaced polyethylene tracheal tube with steel wire tracheal tube, and added electronic vision. Frequency fog-proof visual laryngoscope; laryngeal mask is added as an auxiliary ventilation device.

When astronauts return to Earth in an emergency manner, fluid transfer under weightlessness leads to congestion and edema of the astronauts' head, face, jaw, tongue and nasal cavity, which increases the difficulty of tracheal intubation and ventilation. The application of laryngeal mask for supraglottic ventilation may be the fastest and most effective means of airway opening in the field [27]. According to the characteristics of the two astronauts, the medical team equipped each astronaut with laryngeal masks No. 3 and No. 4.

The medical team trained a lot of simulated dummies to ensure that each medical team member mastered the skills of electronic video anti-fog visual laryngoscope tracheal intubation and laryngeal mask placement ventilation. Each astronaut was equipped with corresponding models of laryngoscope, laryngeal mask and tracheal intubation to ensure the airway opening and ventilation when the astronauts returned in emergency.

\subsubsection{Advanced Life Support}

$i$. Preparation of cardiovascular drugs and fluid channels

Aiming at the changes of cardiovascular, body fluid distribution and water and electrolyte environment when astronauts returned to earth in the middle of their stay in space, the medical team made preparations for medical rescue equipment for several months, and completed the modification of the special first aid box for manned astronauts. Cardiovascular active drugs such as adrenaline and other alpha-and beta-adrenergic receptor agonists and atropine for cardiovascular and humoral changes of astronauts stationed in the medium term are all carried with corresponding doses according to the weight of astronauts in advance, and micro-pumps are carried to ensure the sustained and stable infusion of first-aid drugs.

Two astronauts were equipped with suspended red blood cell blood $1200 \mathrm{ml}$ and plasma $800 \mathrm{ml}$ according to their blood group. In order to prevent the liquid from freezing and maintain the appropriate temperature, a liquid insulating box is equipped, which fully meets the requirements of blood transfusion and transfusion under the pre-cabin emergency medical rescue procedure.

The first aid kit is equipped with advanced and portable medical first aid equipment, such as intra-osseous (IO) system, which can quickly establish the transfusion life passage in one minute. 


\section{ii. Application of Portable Ultrasound}

Ultrasound system is not only an important equipment for clinics of Tiandi Cooperative Telemedicine Association in China, but also undertakes many tasks in emergency disposal after landing of the return capsule. Five-minute rapid ultrasonography can be used to rapidly assess the injury of heart, lung, liver, kidney and cavity organs in astronauts; airway ultrasonography can assess whether the airway of astronauts has changed and obstructed after their mid-term stay in space; because it is difficult to open the vein channel during emergency return, the formation of vein perforation guided by ultrasonography can be achieved. The power is greatly increased. If surgical anesthesia is needed, local anesthesia is a safe choice. Ultrasound-guided nerve block is the most suitable anesthesia method [28].

According to the actual situation, the medical team repeatedly used portable ultrasound (M7 Hand-carried Color Doppler Ultrasound Diagnostic, Mindray) on the return capsule and helicopter rescue platform of the landing site to conduct a large number of live simulation exercises, with an average of 3-5 minutes to complete the heart, lung, liver, kidney, inferior vena cava filling, thoracic cavity, abdomen. Ultrasonography of the cavity and airway.

\subsubsection{Anesthesia Implementation Plan}

On the basis of literature review and characteristic analysis, combined with actual combat, training and drilling, the medical team put forward the following anesthesia scheme after returning to landing.

The safest steps for general anesthesia are: rapid sequence induction under the guidance of ultrasound or after establishing a liquid channel using a bone marrow infusion system, giving general anesthetics (most likely ketamine) with less impact on hemodynamics, using video laryngoscope for tracheal intubation or using laryngeal mask for mechanical ventilation.

For limb or superficial surgery, ultrasound-guided nerve block or local intravenous anesthesia alone can also be combined with sedative drugs.

Small superficial operations can be performed under local intravenous anesthesia.

\section{Discussion}

Over the past 50 years, human manned space missions have been developing continuously. Whether in flight or emergency return phase, emergency resuscitation and anesthesia of astronauts in emergencies must be involved. The emergency resuscitation techniques and anesthesia procedures involved in the emergency medical treatment of astronauts must be managed according to the special problems of astronauts exposed to microgravity. So far, we know little about emergency resuscitation and anesthesia for astronauts under special physiological conditions after long-term and medium-term space missions. Only a few studies have involved airway management after exposure to microgravity and critical care and first aid resuscitation of astronauts. Laryngeal mask is recommended for airway management. So far, no case of emergency cardiopulmonary resuscitation and anesthesia has been reported in the history of human space flight. In view of the particularity of aerospace medical service object, the significance of prevention is obviously greater than that of remedy. Everything should be focused on prevention before it happens. In space expeditions, medical planning inevitably involves some difficult decisions, such as whether the ability to treat catastrophic diseases should be provided or whether the ability to mitigate catastrophic diseases should be provided only from the reality of the mission. In the absence of strong evidence, it is advisable to make worst-case-based choices and solutions. For example: astronauts shock, arrhythmia, difficult intubation, managed by untrained non-medical personnel, medical personnel on board the spacecraft lost their capacity or died.

From "Shenzhou 5" to "Shenzhou 10", the medical team of the main landing site successfully guaranteed all previous space support missions through continuous improvement and optimization of medical security mode. However, Shenzhou 11 is the first manned spacecraft to return in winter in China's manned space history. The two astronauts spent 33 days in orbit, creating a new record of Chinese astronauts' space residence time. These characteristics also put forward higher requirements for the mission of medical rescue and support for astronauts. The altitude of the main landing site is between 1000 and 2100 meters. The annual average temperature is $1-6^{\circ} \mathrm{C}$, the winter average temperature drops from $-14^{\circ} \mathrm{C}$ to $-17^{\circ} \mathrm{C}$ from north to south, and the extreme minimum temperature is $-39^{\circ} \mathrm{C}$. In cold weather, polyethylene tracheal tube is liable to freeze crack and leak; ordinary video laryngoscope is liable to fog under cold conditions, which affects glottis exposure; portable ventilator with lithium battery can not work normally at temperature below $-10 \sim 5^{\circ} \mathrm{C}$. When the astronauts returned to Earth in an emergency manner, the redistribution of body fluids caused by medium-term exposure to microgravity caused congestion and edema of the astronauts' head, face, mandible, tongue and nasal cavity. On the basis of the original pre-cabin rescue and resuscitation, aiming at the various impacts caused by the first mid-term stay of Chinese astronauts in space and the extreme environment of the main landing site, this mission has improved and optimized the previous pre-cabin rescue and resuscitation process and equipment, and formulated an anesthesia rescue plan for the manned space landing site. Combined with simulation training and drilling, the medical security of Shenzhou 11 spacecraft astronauts' main landing site was successfully completed, and valuable experience was accumulated for medical security and anesthesia rescue work of future space exploration missions.

\section{Conclusion}

The Shenzhou 11 Medical Team, aiming at the situation of Chinese astronauts' first medium-term orbital stay, cold weather and complex conditions, on the basis of the original pre-cabin first aid and helicopter first aid platform, through targeted improvement and process optimization, fully meets the effective treatment of astronauts' accidental injuries in cold conditions. Human exploration of space is in the ascendant. China's manned space industry is developing vigorously. With 
the continuous exploration of deep space in the future, astronauts' time on orbit is prolonged. When they return to Earth, they may encounter various new situations, such as sea return and desert return. Astronauts' medical security and anesthesia rescue at the main landing site will also face more problems. Further research and innovation are needed.

\section{Acknowledgements}

This work is supported by The Strategic Support Force Medical Center of The Chinese People's Liberation Army. We thank Professor Yue Maoxing and Dr. Liu Zhiguo for enlightening discussions.

\section{References}

[1] P. D. Hodkinson, R. A. Anderton, B. N. Posselt, et al. An overview of space medicine [J]. British Journal of Anaesthesia, 2017, 119 (S1): i143-i153.

[2] Summers RL, Johnston SL, Marshburn TH, et al. Emergencies in space [J]. Ann Emerg Med, 2005, 46: 177-184.

[3] Matthieu komorowski, Mark R. Campbell. The Bion project and History of Post-Spaceflight Primate Anesthesia [J]. Aerosp Med Hum Perform, 2018, 89 (4): 414-415.

[4] James W, Eugene E, John D. Anesthesia during and after exposure to microgravity [J]. Aviat Space Environ Med, 2004, 571-580.

[5] Norsk P, Asmar A, Damgaard M, et al. Fluid shifts, vaso dilatation and ambulatory blood pressure reduction during long duration space flight [J]. J Physiol, 2015, 593: 573-584.

[6] Watenpaugh DE, Hargens AR. The cardiovascular system in microgravity [M]. Comprehensive physiology. In: Terjung R (ed), 2011.

[7] Liu Ji, Jia Fukun, Song Haifeng, et al. First aid cardiopulmonary resuscitation at the exit of capsule and strategy coping with the "Shenzhou 11 "spacecraft [J]. Chinese Journal of Emergency Medicine, 2018, 27 (8).

[8] Convertino VA, Cooke WH. Evaluation of cardiovascular risks of space flight does not support the NASA Bioastronautics Critical Path Roadmap [J]. Aviat Space Environ Med, 2005, 76: 869-876.

[9] Hamilton DR. Cardiovascular disorders [M]. Principles of clinical medicine for space flight. New York, Springer, 2008, 317-359.

[10] Liu Ji, Jia Fukun, Lou Xiaotong, et al. First aid resuscitation and coping strategy of the main landing site of the "Shenzhou 11 " spacecraft [J]. Chinese Journal of Critical Care Medicine, 2019, 39 (1).

[11] Cooke WH, Ames JE IV, Crossman AA, et al. Nine months in space: Effects on human autonomic cardiovascular regulation [J]. J Appl Physiol, 2000, 89: 1039-1045.

[12] Norsk P, Damgaard M, Petersen L, et al. Vasorelaxation in space [J]. Hypertension, 2006, 47: 69-73.
[13] Buckey JC, Gaffney FA, Lane LD, et al. Central venous pressure in space [J]. New Engl J Med, 1993, 328: 1853-1854.

[14] Hargens AR, Richardson S. Cardiovascular adaptations, fluid shifts, and countermeasures related to space flight [J]. Respir Physiol Neurobiol, 2009, 169 (Suppl 1): S30-S33.

[15] Arbeille P, Kerbeci P, Mattar L, et al. Insufficient flow reduction during LBNP in both splanchnic and lower limb areas is associated with orthostatic intolerance after bedrest $[\mathrm{J}]$. Am J Physiol Heart Circ Physiol, 2008, 295: H1846-254.

[16] Demiot C, Dignat-George F, Fortrat J-O, et al. WISE 2005: Chronic bed rest impairs microcirculatory endothelium in women [J]. Am J Physiol Heart Circ Physiol, 2007, 293: H3159-H3164.

[17] Prisk GK. The lung in space [J]. Clin Chest Med, 2005, 26: 415-438.

[18] Hedenstierna G, Edmark L. Mechanisms of atelectasis in the perioperative period [J]. Best Pract Res Clin Anaesthesiol, 2010, 24: 157-169.

[19] Kirkpatrick AW, Pelosi P, Waele JJD, et al. Clinical review: Intra-abdominal hypertension: Does it influence the physiology of prone ventilation? [J]. Crit Care, 2010, 14: 232.

[20] Williams D, Kuipers A, Mukai C, et al. Acclimation during space flight: Effects on human physiology [J]. CMAJ, 2009, 180: $1317-1323$.

[21] Crucian B, Stowe $\mathrm{R}$, Mehta $\mathrm{S}$, et al. Immune system dysregulation occurs during short duration space flight on board the space shuttle [J]. J Clin Immunol, 2013, 33: 456-465.

[22] Smith SM, Zwart SR, Block G, et al. The nutritional status of astronauts is altered after long-term space flight aboard the International Space Station [J]. J Nutr, 2005, 135: 437-443.

[23] Yue Maoxing, Zou Dewei, Min Qingwang, et al. Medical support and rescue for the First Chinese astronaut in the Landing place $[\mathrm{J}]$. Chinese Journal of Aerospace Medicine, 2003, 14 (4): 193-195.

[24] Liu Zhiguo, Min Qinwang, Jia Fukong, et al. Optimized layout and application of helicopter medical platiform for manned space flight [J]. Chinese Medical Equipment Journal, 2015, 36 (4): $35-36,39$.

[25] Yang Heming, Lou Xiaotong, Song Haifeng, et al. Medical support for astronauts in the main landing field of Shenzhou eleven manned spaceship $[\mathrm{J} / \mathrm{CD}]$. Chin J Hygiene Rescue (Eletronic Edition), 2017, 3 (1): 37-39.

[26] Tan Rong, Gu Jianwen, Li Lu, et al. The specialty and coping strategies of "ShenZhou 11" astronauts's rescue and medical aid [J]. Chinese Journal of Emergency Medicine, 2016, 25 (12): $1325-1328$

[27] Matthieu Komorowski, Sarah Fleming, Mala Mawkin, et al. Anaesthesia in austere environments: literature review and considerations for future space exploration missions $[\mathrm{J}]$. Nature, 2018, 4: 5.

[28] Andrew W, Kirkpatrick, Jeffrey A. Jones, et al. Trauma Sonography for Use in Microgravity [J]. Aviation, Space, and Environmental Medicine, 2007, 78 (4): A38-42. 